\title{
Optimizing Trehalose-Based Quantification of Live Eggs in Potato Cyst Nematodes (Globodera rostochiensis and G. pallida)
}

N. Ebrahimi, Institute for Agricultural and Fisheries Research, Burg. Van Gansberghelaan, Merelbeke 9820, Belgium, and Department of Crop Protection, Faculty of Bio-science Engineering, Ghent University, Coupure links 653, Ghent 9000, Belgium; N. Viaene, Institute for Agricultural and Fisheries Research, and Department of Biology, Faculty of Science, Ghent University; and M. Moens, Institute for Agricultural and Fisheries Research, and Department of Crop Protection, Faculty of Bio-science Engineering, Ghent University

\begin{abstract}
Ebrahimi, N., Viaene, N., and Moens, M. 2015. Optimizing trehalose-based quantification of live eggs in potato cyst nematodes (Globodera rostochiensis and G. pallida). Plant Dis. 99:947-953.

Integrated management of potato cyst nematodes (PCN; Globodera rostochiensis and G. pallida) relies heavily on the determination of cyst population densities in soil as well as the viability of the eggs inside the cysts. This study aimed to optimize a quantitative method to determine the number of viable eggs of PCN based on trehalose present in live eggs. Trehalose was extracted from cysts and from a dilution series of eggs and quantified. More trehalose was detected when cysts were crushed than when left intact. Reaction volumes were adapted to the number of eggs because small reaction volumes hampered an accurate extraction of trehalose. A maximum of $10.5 \mathrm{eggs} / \mu \mathrm{l}$ of reaction volume should be used to obtain a significant linear relationship between detected trehalose content and egg numbers. The sensitivity of the trehalose-based method was evaluated by determining the lowest egg detection limit and was defined

as five viable eggs. The reliability of this method was tested by comparing efficacy with that of two commonly used assays, visual assessment and hatching test. The trehalose-based method gave viability results similar to those of the visual assessment, which is time consuming, requires trained personnel, and can involve some subjectivity. The hatching test identified fewer viable eggs than the other two methods. In addition, the viability of dead eggs (heated and naturally dead) was tested. No false-positive results (dead eggs declared viable) were obtained with the trehalose-based method. The robustness of the test was demonstrated by measuring the viability of eggs of PCN in different experiments repeated in time. The viability assessment method based on trehalose proved to be an objective as well as sensitive, reliable, robust, fast, and cheap technique for assessing the number of viable eggs in PCN cysts.
\end{abstract}

The potato cyst nematodes (PCN) Globodera rostochiensis and G. pallida are the most problematic plant-parasitic nematodes of potato in Europe, causing an annual loss of approximately $\$ 383$ million $(9,10)$. PCN have been reported on all continents (e.g., in Europe [13], Oceania [14,16,25], and the Americas [3,11,22]) where potato crops are grown (19).

Unhatched second-stage juveniles (J2) within the eggs inside of cyst have been found to survive and remain viable for many years in soil, even in the absence of host plants. The long-term survival of PCN seems to be mainly associated with the limited host range of the nematodes as well as the composition of the egg shell and the cyst wall (21). PCN are quarantine organisms in most countries where they can cause yield reduction of potato. However, strict quarantine measures and the effective use of phytosanitary methods combined with other management strategies (e.g., long rotations, application of nematicides, and the use of resistant cultivars) are in place to mitigate the introduction and eventually damage and spread of PCN (13). This is of importance because, once PCN becomes established, protection afforded by the cyst wall and the eggshell reduce the efficacy of many management strategies (e.g., the application of nematicides).

Knowledge of the initial population densities and viability of PCN eggs in fields is required for decision making in an integrated management program. Seinhorst (24) demonstrated that crop yield is strongly influenced by preplant (initial) nematode densities (Pi), which are estimated through soil sampling of the field, followed by extraction of the cysts from the sample and determination of the

Corresponding author: N. Ebrahimi;

E-mail: negin.ebrahimi@ilvo.vlaanderen.be

Accepted for publication 19 December 2014.

http://dx.doi.org/10.1094/PDIS-09-14-0940-RE

(C) 2015 The American Phytopathological Society content of these cysts (sometimes based on a subsample), and expressed as the number of eggs and $\mathrm{J} 2$ (in the cysts) per volume of soil. The damage threshold of PCN is 1 to 2 eggs/g of soil (28). If the cyst content is completely viable, the $\mathrm{Pi}$ is estimated correctly. However, cysts may contain nonviable eggs or J2. In that case, the Pi will be overestimated, leading to needless management efforts. Therefore, information on the viability of eggs and $\mathrm{J} 2$ in the cyst would make the management of PCN more accurate. The viability of encysted eggs is also a critical issue in the interpretation of results of the statutory preplant sampling for seed potato production as well as for certification of other kinds of plants for planting where no viable egg should be present (1). According to the European Union legislation, if PCN are found in a field, no seed potato can be produced in this field; however, ware potato may be grown provided officially approved management practices are applied to suppress the PCN and minimize their spread in the region.

The viability of the cyst content of PCN can be measured by visual assessment based on morphology, Meldola's blue staining followed by microscopic observations, hatching assays, or plant infectivity assays (18). However, the first three aforementioned methods contain an element of subjectivity or are labor intensive and time consuming. van den Elsen et al. (30) reported a qualitative viability test based on the detection of trehalose in live eggs. The assay, however, was not applied to enumerate live eggs. Recently, a quantitative polymerase chain reaction (PCR)-based method in combination with a photoreactive DNA-intercalating dye (propidium monoazide) has been developed for the quantification of viable PCN eggs (4). Compared with this method, a quantitative technique based on trehalose seems to be a feasible and cheaper method.

Trehalose ( $\alpha$-D-glucopyranosyl- $\alpha$-D-glucopyranoside) is a disaccharide present in the perivitelline fluid between the unhatched $\mathrm{J} 2$ and the eggshell. Eggs of G. rostochiensis contained $6.4 \%$ trehalose at a concentration of $0.34 \mathrm{M}(5,20)$. Because of the limited permeability of the lipid layer of the eggshell, the trehalose is retained within the egg. In the absence of a suitable host, PCN survive as $\mathrm{J} 2$ inside the eggs within the cyst. Under the influence of host root 
diffusate, the permeability of the inner lipoprotein membranes of the eggshell increases, the trehalose diffuses out of the eggs, and $\mathbf{J} 2$ hatch from the eggs $(6,7)$. Changes in the permeability of the eggshell upon death of the $\mathrm{J} 2$ embedded in the egg also lead to the diffusion of trehalose out of the eggs. As a consequence, the presence of trehalose in the egg is an indication of the viability of eggs. Assessing the viability of the egg content by estimating the trehalose content of the egg includes the hydrolysis of trehalose into two glucose molecules followed by the detection of glucose.

The ultimate goal of this research was to obtain an optimized method to quantify live eggs and cysts of PCN. We did this based on a previously described trehalose-based method by van den Elsen (30) for the qualitative determination of viability of PCN. Specific questions which were addressed included (i) development of a method to determine the number of viable eggs of PCN in a sample, using the quantity of trehalose present in the eggs, and assessment of its sensitivity; (ii) evaluation of the reliability of this trehalose-based method by comparing it with commonly used viability assays; and (iii) assessment of the robustness of the quantitative trehalosebased method by using cysts of both PCN species.

\section{Materials and Methods}

PCN cultures. Populations of G. rostochiensis (Kruishoutem, East Flanders, Belgium) and G. pallida (Chavornay, Switzerland) were maintained on potato (Solanum tuberosum) 'Désirée'. Potato plants were grown in 3-liter pots filled with a growing medium composed of silver sand, clay powder, and expanded clay granules (4:0.7: 1). In separate pots, the substrate was infested with 5 eggs and $\mathbf{J} 2$ per cubic centimeter of soil and cultures were maintained in a greenhouse ( 20 to $25^{\circ} \mathrm{C}$ and $16 \mathrm{~h}$ of light) and watered regularly. After 16 weeks, newly formed cysts were extracted from the substrate using a Seinhorst elutriator (23). Cysts were kept for at least 4 months at $4^{\circ} \mathrm{C}$ to overcome diapause before use in experiments. The average number of eggs per cyst was based on 20 cysts of each PCN species. Cysts were placed in a drop of water in a petri dish and crushed. The released eggs were rinsed into a beaker over a $60-\mu \mathrm{m}$ sieve and the volume adjusted to $50 \mathrm{ml}$. The number of eggs and $\mathrm{J} 2$ in three $3-\mathrm{ml}$ subsamples was determined using a microscope $(\times 100)$.

Hatching assay. The viability of the cultured eggs that were used in subsequent experiments was assessed with a hatching assay. The hatching assay was also used as a reference to evaluate the results (number of viable eggs and J2) obtained with the trehalose method (see below). For hatching assays, potato root diffusate (PRD) was collected from Désirée potato following the protocol described by Turner et al. (27). A potato tuber was planted in a 2-liter pot filled with a sterilized sandy soil. After 4 weeks, the soil was saturated with water and then an additional $100 \mathrm{ml}$ of water was added. The solution draining from the pot was collected and passed through the pot again. The second root diffusate was filtered (Munktell filter paper number 2601, $150 \mathrm{~mm}$, basis weight $40 \mathrm{~g} \mathrm{~m}^{-2}$; Munktell Filter AB, Falun, Sweden) to remove soil particles and stored at $-20^{\circ} \mathrm{C}$ for later use.

Four replicates of 20 cysts of G. rostochiensis or G. pallida were soaked in water for $24 \mathrm{~h}$ before being exposed to PRD. The center of the lid of a 1.5-ml Eppendorf tube was replaced with a $250-\mu \mathrm{m}$ nylon mesh sieve and the constricted end of the tube was cut off, and the tube was placed upside down in a 1-cm-diameter plastic vial. Intact cysts were transferred to the tube and $2 \mathrm{ml}$ of the PRD was added to the vial, just touching the sieve. Samples were maintained at $21^{\circ} \mathrm{C}$ in a completely randomized design. The number of hatched $\mathrm{J} 2$ which moved through the sieve into the vial was counted at weekly intervals over a period of 14 weeks and the PRD was renewed at every counting event. After 14 weeks, cysts were crushed and the number of viable unhatched $\mathbf{J} 2$ inside the cysts was counted. This number, together with the number of hatched $\mathbf{J} 2$, allowed for the calculation of the percentage hatch of eggs. The same procedure was applied in further experiments where hatching in PRD was used as a reference test, although with different numbers of cysts and for varying periods of time.

Visual assessment of viability. For the visual determination of the viability of cultured eggs, five replicates of 20 cysts of G. rostochiensis and of G. pallida were soaked in tap water for $24 \mathrm{~h}$ at $21^{\circ} \mathrm{C}$ before releasing the eggs inside the cysts by crushing (see above). The volume of the egg suspension obtained from each replicate (20 cysts) was increased to $50 \mathrm{ml}$. The number of viable eggs in the suspension was determined based on three 3-ml subsamples using a microscope $(\times 100)$. Damaged and empty eggs with a nonsmooth eggshell and shriveled, disintegrated $\mathbf{J} 2$ with no clear lip region or stylet were considered dead, as described in the European and Mediterranean Plant Protection Organization protocol PM7/41 $(2,18)$. The visual assessment of viability of eggs of both PCN species was performed twice.

Quantification of trehalose in cysts and the effect of crushing cysts on the amount of trehalose detected. Trehalose in eggs of noncrushed cysts of G. rostochiensis or G. pallida was measured using a detection kit (K-TREH 01/09; Megazyme International Ireland Ltd., Wicklow, Ireland) following the protocol of van den Elsen et al. (30). Single or multiple cysts of G. rostochiensis or G. pallida were transferred to 0.2-ml Eppendorf tubes containing $10 \mu \mathrm{l}$ of MilliQ water. Blank samples containing $10 \mu l$ of MilliQ water without any cyst were also included in the experiment. Samples were incubated for $30 \mathrm{~min}$ at $99^{\circ} \mathrm{C}$ in a thermomixer (HLC, MHR 13; Pforzheim, Germany) at $300 \mathrm{rpm}$ to extract trehalose. Samples were cooled to room temperature before $12 \mu \mathrm{l}$ of MilliQ water, $2 \mu \mathrm{l}$ of imidazole, $1 \mu \mathrm{l}$ of NADP + ATP solution, and $0.2 \mu$ of hexokinase and glucose-6phosphate dehydrogenase ( 0.425 and $0.212 \mathrm{U} / \mu \mathrm{l}$, respectively) were added to each sample for a total reaction volume of $26.2 \mu 1$. Samples were homogenized using a vortex and centrifuged at 12,000 rpm for $20 \mathrm{~s}$. Then, the samples were incubated for $5 \mathrm{~min}$ at room temperature before the absorbance of NADPH at $\lambda=340 \mathrm{~nm}\left(\mathrm{~A}_{1}\right)$ was measured using a NanoDrop (ND-1000; Isogen, The Netherlands). Trehalase $(1 \mu \mathrm{l})$ was added and samples were again homogenized using a vortex, centrifuged at $12,000 \mathrm{rpm}$ for $20 \mathrm{~s}$, and incubated at room temperature for $5 \mathrm{~min}$ before the absorbance of light at $\lambda=340 \mathrm{~nm}$ by NADPH was measured $\left(A_{2}\right)$. Subsequently, the difference in the absorbance of NADPH was calculated $\left(\mathrm{A}_{2}-\mathrm{A}_{1}\right)$. The difference in the absorbance of the blank sample $\left(\Delta \mathrm{A}_{\text {blank }}=\mathrm{A} 2-\mathrm{A} 1\right)$ was subtracted from the difference in absorbance of the samples with cysts $\left(\Delta \mathrm{A}_{\text {trehalose }}=\left(\mathrm{A}_{2}-\mathrm{A}_{1}\right)-\Delta \mathrm{A}_{\text {blank }}\right)$. The concentration of trehalose was calculated as follows:

$$
[\text { Trehalose }]=\Delta \mathrm{A}_{\text {trehalose }} \times\left(\mathrm{MW}_{\text {trehalose }} / \varepsilon \times d \times 2\right)
$$

where MW is the molecular weight of trehalose $\left(\mathrm{g} \mathrm{mol}^{-1}\right), \varepsilon$ is the extinction coefficient of NADPH at $340 \mathrm{~nm}, d$ is light path $(\mathrm{cm})$, and 2 represents 2 molecules of D-glucose released from each hydrolyzed molecule of trehalose. The amount of NADPH formed is related to the amount of D-glucose and, thus, with twice the amount of trehalose. Eventually, the amount of trehalose in the whole sample was calculated by multiplying the concentration of trehalose by the reaction volume.

The relationship between the number of viable cysts and the trehalose detected after extraction with the above procedure was determined by quantifying the trehalose content of $1,5,10,15$, and 20 cysts of $G$. rostochiensis and of $G$. pallida in reaction volumes of 26.2, 52.4, 78.6, and 104.8 $\mu \mathrm{l}$. Different reaction volumes were chosen to examine the possible effect of the amount of the reaction volume on the quantity of trehalose detected. For every reaction volume, samples were arranged in a randomized complete block design. Each combination of the cyst number and the reaction volume had 10 replicates. For volumes where results indicated a linear association between the number of eggs and the trehalose content, the best-fit linear regression line was calculated. These experiments were conducted three times $(n=30)$. In the case of single cysts, the number of eggs was counted to correlate the measured quantity of trehalose to the number of viable eggs (data not shown).

The effect of crushing cysts on the quantity of trehalose detected was examined in another experiment. Ten batches of 1 crushed or noncrushed cyst of each of the PCN were prepared prior to trehalose extraction in a reaction volume of $26.2 \mu \mathrm{l}$ (as described above). This test was also performed three times $(n=30)$.

Trehalose level in nonviable cyst samples. Because of the quarantine status of PCN, the viability test might also be useful to determine the presence or absence of viable eggs in a sample. To define 
the threshold below which a trehalose signal cannot be detected in viable eggs, PCN cysts with nonviable eggs (i.e., without trehalose) were used. Nonviable cysts were obtained artificially and from the field. Cultured viable cysts of G. rostochiensis and G. pallida were presoaked in water for $24 \mathrm{~h}$ before being killed by incubation at $99^{\circ} \mathrm{C}$ in MilliQ water for $30 \mathrm{~min}$ (30) and replacing the supernatant (that contains the released trehalose) by MilliQ water. After incubation at room temperature for 2 days, the supernatant was again discarded and the cysts were washed four times with MilliQ water. MilliQ water was added to the rinsed cysts, which were then incubated at room temperature for another day. Finally, the supernatant was discarded and the cysts were rinsed six times with MilliQ water. The viability of 20 of these cysts of each PCN species was checked for each individual cyst using the trehalose-based method (see above). This was repeated with another batch of 20 cysts of both PCN species $(n=40)$. Additionally, naturally dead cysts of G. rostochiensis, collected from two different fields and stored in the lab at room temperature for more than 10 years, were examined. In all, 20 cysts, 10 from each field, were used separately for the trehalose-based method. This test was performed twice $(n=20)$. The nonviability of cysts from the field populations and of the heat-killed cysts was confirmed using subsamples of 20 cysts of each category. Both the visual method and the hatching assay (10 weeks) were performed on individual cysts in repeated experiments $(n=40)$.

Detection threshold of the trehalose-based method. The sensitivity of the trehalose-based method was evaluated by determining the lowest number of viable eggs or cysts that can be detected. Four different experiments were conducted. In experiments 1 and 3 , the trehalose content of 1, 2, 3, 4, 5, and 10 eggs (in a reaction volume of $26.2 \mu \mathrm{l}$ ) and of $1,5,10,15$, and 20 cysts (in a reaction volume of $104.8 \mu \mathrm{l}$ ) was measured, respectively (Table 1). In experiment 4 , to examine the detection of viable cysts of $G$. rostochiensis mixed with nonviable cysts, viable cysts were mixed with heat-killed, nonviable cysts in different proportions $(1: 19,5: 15,10: 10,15: 10$, and 20:10 viable/nonviable cysts) each in a reaction volume of $104.8 \mu \mathrm{l}$ (Table 1). In experiment 2, heat-killed cysts were crushed and nonviable eggs were mixed with viable eggs in proportions of 5 viable eggs mixed with $5,10,15$, and 20 nonviable eggs, as well as 10 viable eggs also mixed with $5,10,15$, and 20 nonviable eggs (Table 1). Subsequently, the trehalose content was extracted and quantified in a reaction volume of $26.2 \mu \mathrm{l}$. Experiments were replicated 10 times and experiments were conducted three times $(n=$ 30). The same four experiments were performed with cysts and eggs of $G$. pallida with the same level of replication and experimental repeats (Table 1).

Quantification of trehalose in eggs and construction of a standard curve. The quantity of trehalose released from a cyst depends on the number and viability of eggs in the cyst. Hence, to construct a correlation between the number of viable eggs and the trehalose content (a standard curve), the amount of trehalose was determined in a dilution series of eggs. Cysts from cultures of G. rostochiensis and G. pallida were crushed in MilliQ water to obtain a stock egg suspension. The number of viable eggs per unit volume was determined based on four 3-ml subsamples, as described above. A dilution series of 100, 200,300,400,500,600, 700, 800, and 900 viable eggs of both $G$. pallida and G. rostochiensis was prepared. The trehalose contents of the eggs in these dilution series were determined in reaction volumes of 26.2, 52.4, 78.6, and $104.8 \mu$ l. For each reaction volume (treatment), samples were arranged in a randomized complete block design with 10 replicates and the whole experiment was conducted four times for $G$. rostochiensis $(n=40)$ and twice for $G$. pallida $(n=20)$. For volumes where results indicated a linear association between the number of eggs and the trehalose content, the best-fit linear regression line was calculated.

Reliability and robustness of the trehalose-based method. The reliability of the trehalose-based method was tested by comparing its efficacy with visual assessment and the hatching assay using individual cysts. For the visual assessment, 20 individual cysts were crushed separately, as described above. The total percentage of viable eggs in each cyst was determined. For the trehalose-based method, another 20 individual cysts were crushed and eggs were released. Then, trehalose was extracted from all eggs obtained from one cyst in

Table 1. Sensitivity of the trehalose-based method ${ }^{\mathrm{a}}$

\begin{tabular}{|c|c|c|c|c|c|c|c|}
\hline \multirow[b]{2}{*}{ Experiment } & \multirow[b]{2}{*}{ Viable eggs } & \multirow[b]{2}{*}{ Nonviable eggs } & \multirow[b]{2}{*}{ Viable cysts } & \multirow[b]{2}{*}{ Nonviable cysts } & \multirow[b]{2}{*}{ Reaction volume $(\mu \mathrm{l})$} & \multicolumn{2}{|c|}{$\left(\mathbf{A}_{2}-\mathbf{A}_{1}\right)-\Delta \mathbf{A}_{\text {blank }}{ }^{b}$} \\
\hline & & & & & & G. rostochiensis) & G. pallida \\
\hline 1 & 1 & & & & 26.2 & $0.0010 \pm 0.0012 *$ & $0.0015 \pm 0.0010^{*}$ \\
\hline 1 & 2 & & & & 26.2 & $0.0018 \pm 0.0011^{*}$ & $0.0015 \pm 0.0009 *$ \\
\hline 1 & 3 & & & & 26.2 & $0.0021 \pm 0.0022 *$ & $0.0021 \pm 0.0015^{*}$ \\
\hline 1 & 4 & & & & 26.2 & $0.0020 \pm 0.0012^{*}$ & $0.0022 \pm 0.0012 *$ \\
\hline 1 & 5 & & & & 26.2 & $0.0104 \pm 0.0007$ & $0.0104 \pm 0.0006$ \\
\hline 1 & 10 & & & & 26.2 & $0.0126 \pm 0.0031$ & $0.0129 \pm 0.0029$ \\
\hline 2 & 5 & 5 & & & 26.2 & $0.0106 \pm 0.0006$ & $0.0106 \pm 0.0005$ \\
\hline 2 & 5 & 10 & & & 26.2 & $0.0107 \pm 0.0009$ & $0.0106 \pm 0.0008$ \\
\hline 2 & 5 & 15 & & & 26.2 & $0.0104 \pm 0.0005$ & $0.0107 \pm 0.0006$ \\
\hline 2 & 5 & 20 & & & 26.2 & $0.0105 \pm 0.0006$ & $0.0105 \pm 0.0007$ \\
\hline 2 & 10 & 5 & & & 26.2 & $0.0119 \pm 0.0010$ & $0.0118 \pm 0.0009$ \\
\hline 2 & 10 & 10 & & & 26.2 & $0.0122 \pm 0.0021$ & $0.0125 \pm 0.0020$ \\
\hline 2 & 10 & 15 & & & 26.2 & $0.0125 \pm 0.0027$ & $0.0126 \pm 0.0023$ \\
\hline 2 & 10 & 20 & & & 26.2 & $0.0124 \pm 0.0025$ & $0.0123 \pm 0.0025$ \\
\hline 3 & & & 1 & & 104.8 & $0.2811 \pm 0.0781$ & $0.2900 \pm 0.0812$ \\
\hline 3 & & & 5 & & 104.8 & $0.4921 \pm 0.2123$ & $0.4012 \pm 0.2105$ \\
\hline 3 & & & 10 & & 104.8 & $0.3895 \pm 0.1654$ & $0.4184 \pm 0.3257$ \\
\hline 3 & & & 15 & & 104.8 & $0.4813 \pm 0.3112$ & $0.4956 \pm 0.2987$ \\
\hline 3 & & & 20 & & 104.8 & $0.4914 \pm 0.1839$ & $0.4981 \pm 0.2845$ \\
\hline 4 & & & 1 & 19 & 104.8 & $0.2031 \pm 0.1102$ & $0.2952 \pm 0.0798$ \\
\hline 4 & & & 5 & 15 & 104.8 & $0.2974 \pm 0.1014$ & $0.4017 \pm 0.1985$ \\
\hline 4 & & & 10 & 10 & 104.8 & $0.3945 \pm 0.2679$ & $0.4221 \pm 0.3012$ \\
\hline 4 & & & 15 & 10 & 104.8 & $0.4854 \pm 0.2134$ & $0.4890 \pm 0.1983$ \\
\hline 4 & & & 20 & 10 & 104.8 & $0.5098 \pm 0.2387$ & $0.4075 \pm 0.2965$ \\
\hline
\end{tabular}

a Trehalose concentration (mean \pm standard deviation) detected in viable eggs or cysts of G. rostochiensis and G. pallida alone or mixed with nonviable eggs or cysts in different proportions, each containing 30 replicates.

${ }^{\mathrm{b}} \mathrm{A}_{2}-\mathrm{A}_{1}=$ difference in absorbance of NADPH at $\lambda=340 \mathrm{~nm}$ measured in series 1 to $4 ; \Delta \mathrm{A}_{\text {blank }}=\mathrm{A}-\mathrm{A}_{1}=$ difference in absorbance of NADPH at $\lambda=340 \mathrm{~nm}$ measured in a blank sample without cysts or eggs; * indicates below the threshold value for PCN viability $(0.008)$. 
a reaction volume of $78.6 \mu \mathrm{l}$, as described above. The data on trehalose content were converted to the number of eggs based on the equation of the standard curve. The viability of 20 individual cysts was also assessed by determining the hatching percentage after a 10-week exposure of each cyst to the PRD. This experiment was performed twice for both PCN $(n=40)$.

Data analysis. For the construction of the standard curve, linear regressions were performed to assess the relationship between the numbers of cysts or eggs and trehalose content, after assumptions of linear regression were tested. The regression lines obtained from the repeated tests were compared with analysis of covariance. When no significant differences were found among the intercept and the slopes of the regression lines, the data of the repeated tests were combined to construct a standard curve. Data of the trehalose content of crushed and noncrushed cysts were subjected to a $t$ test. The percentage of viable eggs measured by each of the three viability assessment methods was subjected to analysis of variance (ANOVA) to determine the effect of the method on the viability assessment, after assumptions of ANOVA were tested. The differences among the viability assays were analyzed with Tukey's honestly significant difference tests and differences were considered significant at $P<0.05$. All the analyses were performed using STATISTICA_9 software (Statsoft, Tulsa, OK).

\section{Results}

Viability of cultured cysts. The average number of eggs per cyst of G. rostochiensis and G. pallida was $435 \pm 46$ and $418 \pm 51$ eggs, respectively. The final percentage hatch after 14 weeks exposure to PRD was $99 \%$ for both G. pallida and G. rostochiensis. Percentage hatch of $\mathbf{J} 2$ from eggs in cysts of $G$. rostochiensis and G. pallida over 3 weeks was $49 \pm 1.6$ and $37 \pm 2.5 \%$, respectively. After 8 weeks, a period often applied in diagnostic laboratories, the percentage hatch of $\mathrm{J} 2$ for $G$. rostochiensis and G. pallida had reached $84 \pm 1.3$ and $84 \pm 1.5 \%$, respectively.

Quantification of trehalose in cysts and the effect of crushing cysts on the amount of trehalose detected. There was no increase in the detected amount of trehalose with increasing number for cysts of both PCN species when the reaction volume was 26.2, 52.4, or $78.6 \mu$ l (Fig. 1A and C). Similar results were obtained in the repeated tests (data not shown). However, in a reaction volume of $104.8 \mu \mathrm{l}$, the concentration of trehalose increased linearly with increasing numbers of cysts of $G$. rostochiensis and $G$. pallida $(P=0.001$ and 0.01 , respectively; Fig. 1A and $\mathrm{C}$ ). Three regression lines were obtained from the three repeated tests with $G$. rostochiensis cysts in the reaction volume of $104.8 \mu \mathrm{l}\left(y=0.0801 x+2.32, R^{2}=0.97\right.$, $P=0.001 ; y=0.0481 x+2.12, R^{2}=0.85, P=0.04$; and $y=$ $\left.0.0257 x+2.54, R^{2}=0.81, P=0.01\right)$. The slope and intercept of these regression lines were different $(P<0.05$; Fig. 1B); therefore, the data of the three repeated tests were not combined. The same was observed for G. pallida, with the three regression lines $(y=0.0381 x+$ $2.59, R^{2}=0.89, P=0.01 ; y=0.0521 x+2.02, R^{2}=0.72, P=0.04$; and $\left.y=0.0213 x+2.36, R^{2}=0.30, P=0.11\right)$ also being significantly different $(P<0.05$; Fig. 1D). The results of the trehalose content of different numbers of both PCN cysts revealed that only in high reaction volumes was a linear relationship between the number of cysts and the trehalose content achieved. However, this relationship varied among three repeated tests.

The quantity of trehalose released from a single noncrushed and crushed cyst was $2.35 \pm 0.26$ and $2.85 \pm 0.016 \mu \mathrm{g}$, respectively, for $G$. rostochiensis and $2.00 \pm 0.61$ and $3.12 \pm 0.12 \mu \mathrm{g}$, respectively, for G. pallida (Fig. 2). Crushing cysts resulted in higher trehalose concentrations than extracting trehalose from noncrushed cysts for G. rostochiensis and $G$. pallida $(F=39.56, \mathrm{df}=1, P=0.001$ and $F=15.28, \mathrm{df}=1, P=0.001$, respectively).
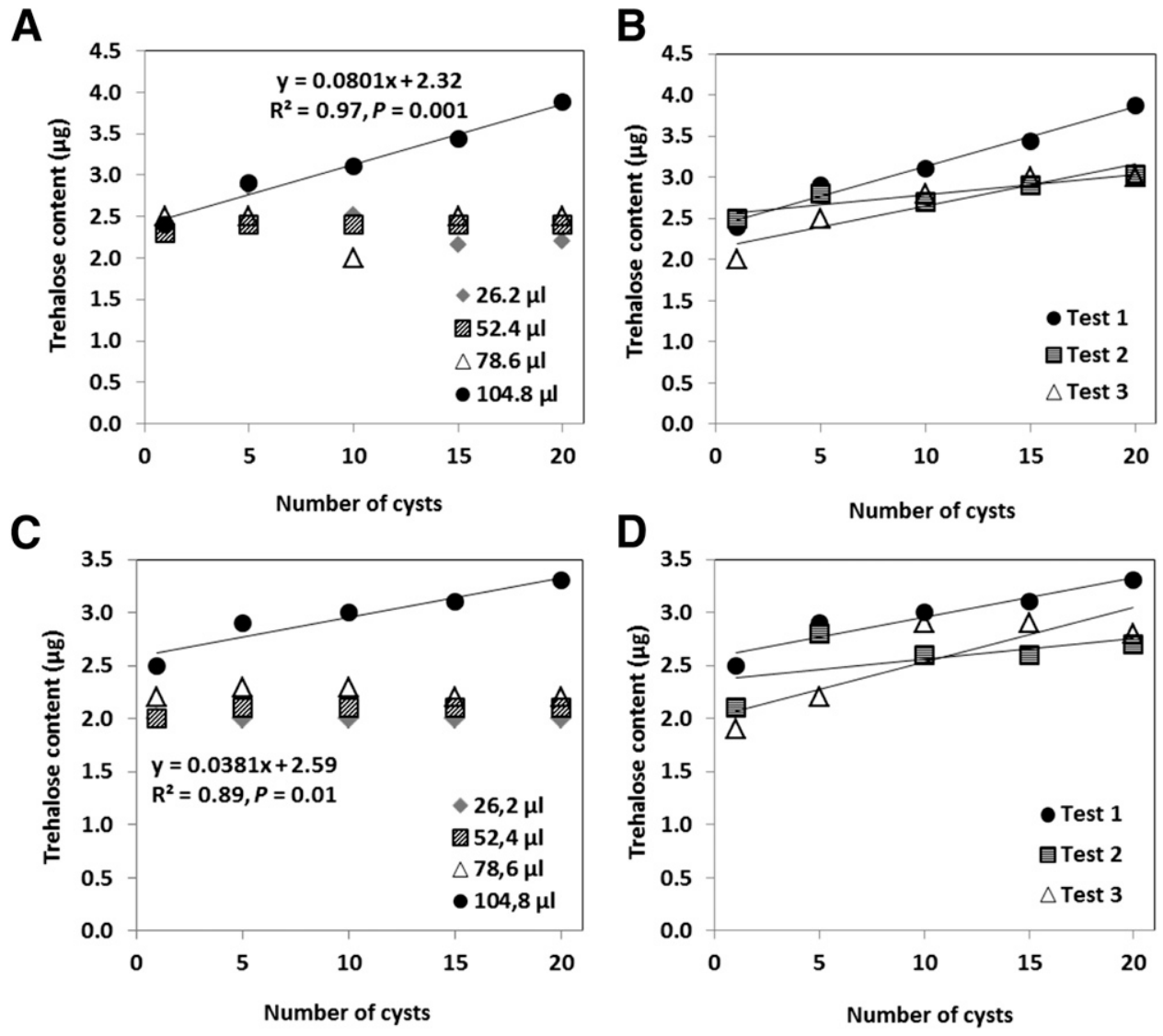

Fig. 1. Relationship between the numbers of noncrushed cysts and the detected amount of trehalose in different reaction volumes for A, Globodera rostochiensis and C, G. pallida (obtained from one test) and in a reaction volume of $104.8 \mu \mathrm{l}$ in three repeated tests for B, G. rostochiensis and $\mathbf{D}, \mathbf{G}$. pallida. The fitted linear regression line corresponds to the relationship between the number of cysts and measured trehalose content in a reaction volume of $104.8 \mu \mathrm{l}$. Each data point is the mean of 10 replicates. 
Trehalose level in nonviable cyst samples. Both heat-treated cysts and old cysts collected from the field were classified as nonviable by both visual assessment $(0 \%)$ and hatching assay $(0 \%)$. The $\Delta \mathrm{A}_{\text {trehalose }}$ for heat-treated cysts was $0.004 \pm 0.002(n=40)$. The $\Delta \mathrm{A}_{\text {trehalose }}$ for old $(=$ dead $)$ cysts originating from the field was $0.005 \pm 0.002(n=20)$. The results for the repeat of the test were similar and the value for $\Delta \mathrm{A}_{\text {trehalose }}$ varied between 0 and 0.008 in both tests. Based on these results, the threshold value for PCN viability was determined as $\Delta \mathrm{A}_{\text {trehalose }}=0.008$; below this value, cysts will be considered nonviable.

Detection threshold of the trehalose-based method. In experiment 1 , only 5 and 10 eggs gave a trehalose signal above the threshold level $\left(\Delta \mathrm{A}_{\text {trehalose }}=0.008\right)$ for both PCN species (Table 1$)$. In experiment 3 , a trehalose signal above the threshold level was detected in all samples containing 1 to 20 cysts. In experiments 2 and 4 , viable eggs or viable cysts of both species were always detected despite the presence of nonviable eggs or nonviable cysts with a $\Delta \mathrm{A}_{\text {trehalose }}$ above the viability threshold. The exception was for $G$. rostochiensis cysts, where less trehalose was detected from

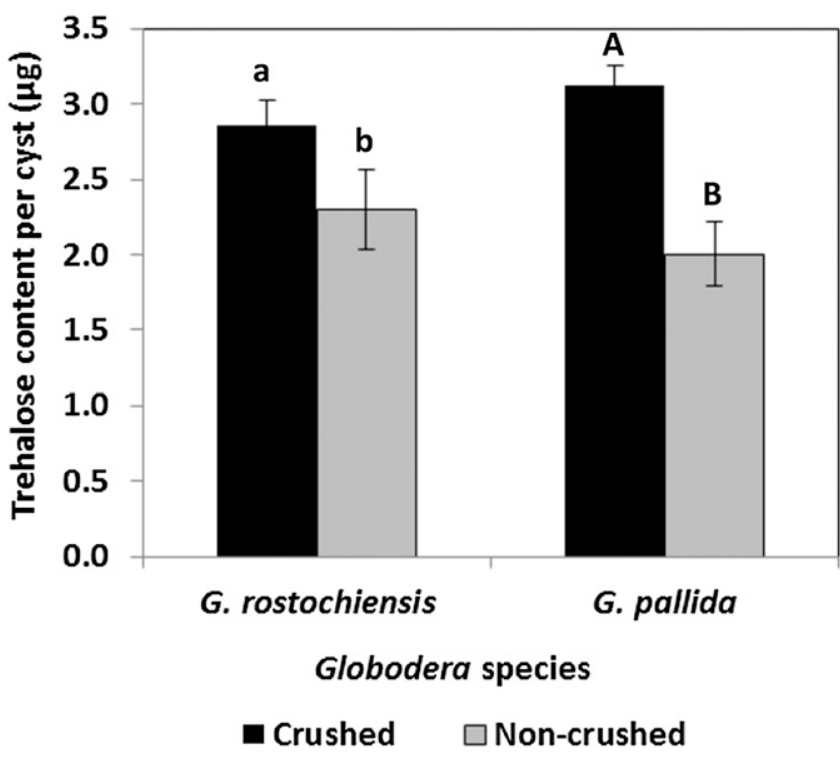

Fig. 2. Trehalose detected in a crushed and noncrushed cyst of Globodera rostochiensis and G. pallida in a reaction volume of $26.2 \mu \mathrm{l}$. Values are the means of the combined data of three tests $(n=30)$. Bars marked with a different lower- or uppercase letter are significantly different according to the $t$ test $(P<0.05)$. Error bars represent the standard deviation of the mean
5 viable cysts mixed with 15 nonviable cysts $(P=0.01)$. This same trend was not observed for G. pallida cysts (Table 1).

Quantification of trehalose in PCN eggs and construction of a standard curve. Extraction of trehalose from eggs of $G$. rostochiensis in a reaction volume of $26.2 \mu \mathrm{l}$ resulted in increasing trehalose content up to 300 eggs (11.4 eggs/ $\mu$ l reaction volume); the trehalose detected in samples with more eggs was approximately constant, independent of the number of eggs (Fig. 3A). When a reaction volume of $52.4 \mu \mathrm{l}$ was used, this plateau phase was reached at approximately 500 eggs, or $9.5 \mathrm{eggs} / \mu 1$ of reaction volume. In reaction volumes of 78.6 and $104.8 \mu \mathrm{l}$, a linear relationship between the number of eggs and trehalose content was observed $(P<0.05)$. However, the regression based on the reaction volume of $78.6 \mu$ l gave the best fit $\left(R^{2}=0.96, P=0.001\right.$; Fig. $\left.3 \mathrm{~A}\right)$. Four regression lines were obtained from the four repeated tests with $G$. rostochiensis eggs $\left(y=0.006 x+0.65, R^{2}=0.96, P=0.001 ; y=0.006 x+0.58, R^{2}=\right.$ $0.96, P=0.001 ; y=0.007 x+0.55, R^{2}=0.95, P=0.002$; and $y=$ $\left.0.006 x+0.59, R^{2}=0.96, P=0.001\right)$. There was no significant difference between these regression lines (data not shown). Therefore, the data of the repeated tests for $G$. rostochiensis were combined to construct a standard curve described by the equation $y=0.006 x+0.62$, where $y=$ the trehalose content of the eggs and $x=$ the number of viable eggs (Fig. 3A). A similar relationship was observed between the trehalose content of $G$. pallida eggs and density in a reaction volume of $78.6 \mu \mathrm{l}$ and was described by $y=0.007 x+0.22\left(R^{2}=0.98, P<\right.$ 0.001 ; Fig. 3B). This equation was also obtained from the combination of data obtained from two repeated tests. Comparing the slopes and the intercepts of the two regression lines for G. pallida $(y=$ $0.007 x+0.22, R^{2}=0.98, P<0.001$ and $y=0.007 x+0.21, R^{2}=$ $0.98, P<0.001)$ also showed no significant difference.

Reliability and robustness of the trehalose-based method. Significant differences were found between the methods for assessing egg viability of cultured cysts of $G$. rostochiensis $(F=5.68, \mathrm{df}=$ $2, P<0.05)$. More eggs were classified viable when assessed visually $(87.9 \%)$ or using the trehalose-based method $(86.8 \%)$ than with the hatching assay (83.1\%) (Fig. 4). Similar results were obtained with cysts of G. pallida: no differences were found between the visual assessment and the trehalose method when assessing the viability of cultured cysts ( 88 and $86.1 \%$, respectively), whereas the hatching test identified fewer eggs as viable $(84.0 \%)(F=6.24, \mathrm{df}=2, P=0.001)$ (Fig. 4).

\section{Discussion}

Our study showed that the trehalose content of eggs of PCN can be quantified and used to determine the number of viable eggs in a sample. The determination of egg viability of both G. rostochiensis and G. pallida using the trehalose-based method yielded similar results. Moreover, similar data were obtained from different repeated
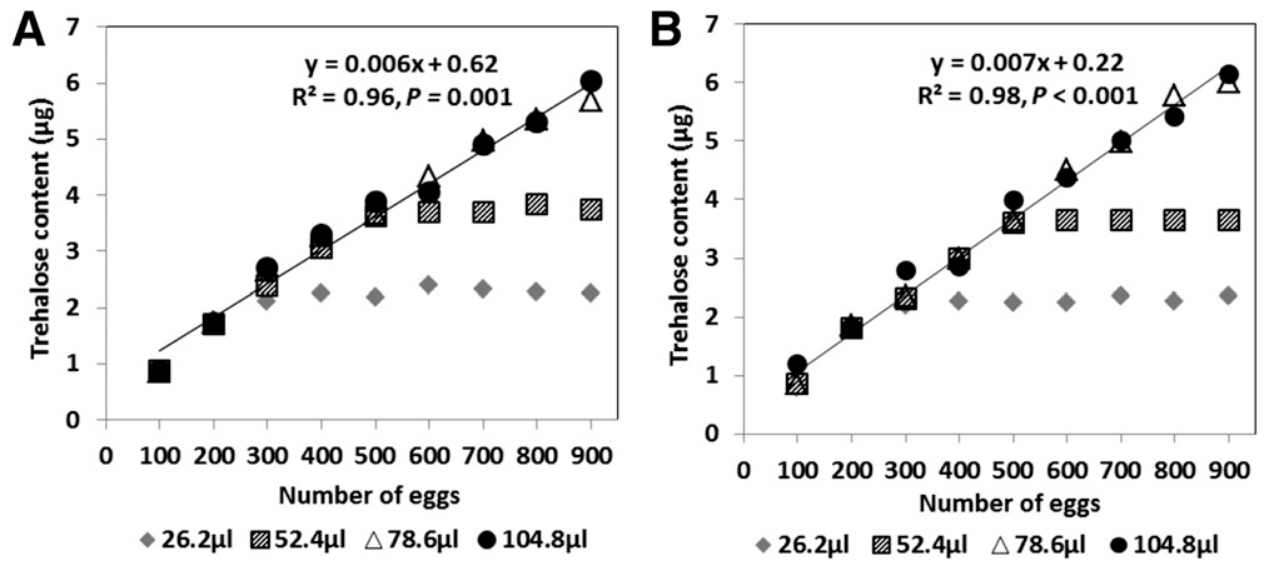

Fig. 3. Relationship between the number of viable eggs of A, Globodera rostochiensis and B, G. pallida and measured trehalose in different reaction volumes. Values are the means of combined data of four repeated experiments $(n=40)$ for $G$. rostochiensis and two repeated experiments $(n=20)$ for $G$. pallida. The fitted linear regression line corresponds to the relationship between the number of eggs and measured trehalose content in a reaction volume of $78.6 \mu$ l. 
experiments for both species. Therefore, it can be concluded that the trehalose-based method is a robust tool to determine the viability of PCN species eggs.

More trehalose was detected in crushed cysts than in intact cysts. Part of the trehalose might have been locked up inside the cysts and unavailable for the reaction with trehalase, or perhaps part of the glucose molecules were retained within the cysts and, therefore, unable to diffuse into the reaction solution. Our results showed that the difference in trehalose detected in a crushed and a noncrushed cyst was 0.55 and $1.12 \mu \mathrm{g}$ for G. rostochiensis and G. pallida, respectively, corresponding with about 50 and 100 live eggs per cyst. Hence, to attain more precise numbers of viable eggs of PCN present in a sample, it is recommended to crush the cysts to release eggs prior to the trehalose extraction. If not, one will underestimate the population density of viable PCN eggs and, consequently, the threat of damage caused by these nematodes.

Because the quantification of viable eggs is based on an enzymatic reaction, the reaction volume of the kit (K-TREH 01/09), including the enzyme, needs to be adapted to the substrate concentration (i.e., the number of viable eggs). We observed that about 150 eggs can be retained in $1 \mu$ l of solution but an accurate quantification of the amount of trehalose is not possible when the number of viable eggs per microliter of reaction solution exceeds approximately 10.5 eggs. The insufficient detection of trehalose when there are more than $10.5 \mathrm{eggs} / \mu \mathrm{l}$ of reaction can be caused by too low a concentration of trehalase needed to hydrolyze the trehalose present in all the eggs to D-glucose, or the inhibition of the trehalase activity due to biochemical changes in the reaction solution. It has been shown that trehalase is inactivated in a suboptimal $\mathrm{pH}$ and temperature (8). Nevertheless, regarding the experimental set up, the effect of temperature cannot be accounted for it.

The threshold value for egg viability established in our experiment was $\Delta \mathrm{A}_{\text {trehalose }}=0.008$, whereas van den Elsen et al. (30) presented a threshold value of 0.0094 for a nonviable PCN population. Additionally, based on the trehalose signal obtained from dead cysts, we defined the detection limit as five viable eggs. van den Elsen et al. (30) showed that, in the case of field populations, the detection limit is 10 viable PCN eggs in a background of 25 nonviable PCN

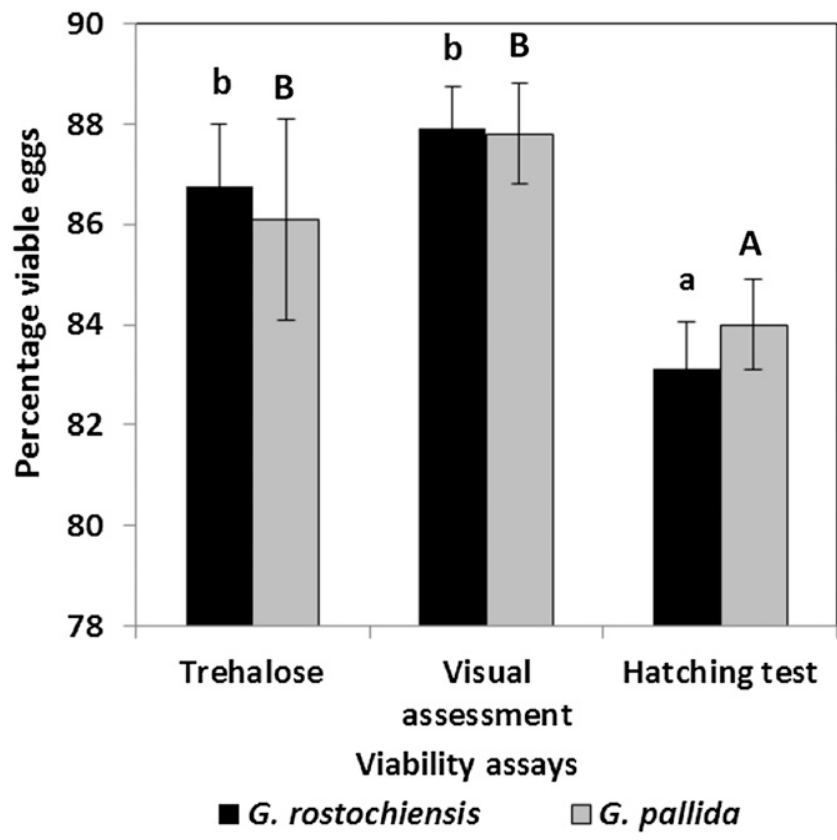

Fig. 4. Comparison of three methods (trehalose-based method, visual assessment, and hatching assay) to measure the viability of eggs of Globodera rostochiensis or G. pallida. Bars are the means of combined data of two experiments and error bars represent the standard errors of the mean $(n=40)$. Bars within a nematode species followed by a different letter (lowercase for $G$. rostochiensis or uppercase for G. pallida) are significantly different according to Tukey's honestly significant difference test $(P<0.05)$. cysts. The number of eggs in young PCN cysts in the field varies, on average, between 100 and 450 eggs; therefore, 5 eggs is an acceptable threshold and the trehalose method can be considered to be very sensitive. However, when dealing with statutory samples where no live cysts should be present, or when evaluating disinfestation or other control measures where $100 \%$ nonviability is required, this threshold should be kept in mind. Nevertheless, other viability assays have similar limitations. Therefore, the restrictions of each viability assay should be considered when selecting a method for a specific purpose.

Our study showed that the presence of nonviable eggs in the sample does not reduce the detection of viable eggs, which was the case for both PCN species. This finding makes the trehalose-based method a sensitive technique when assessing viability of encysted eggs from fields. However, it should be noted that this was not always the case when using cysts instead of eggs. In two of the three repeated experiments, 5 viable cysts of $G$. rostochiensis in a background of 15 nonviable ones yielded less trehalose than 5 viable cysts alone, thereby influencing the sensitivity of the trehalose method. Yet, with the other proportions of dead and viable cysts of $G$. rostochiensis and with all cyst combinations of $G$. pallida, the presence of nonviable cysts did not influence the outcome of the trehalose method. More investigation is needed to address this difference. Nevertheless, this observation with a combination of viable and nonviable cysts again recommends the detection of trehalose in eggs freed from cysts.

In accordance with van den Elsen et al. (30), we found a relationship between the egg viability and the trehalose content. We constructed a standard curve for both PCN species to have a precise correlation between the number of viable eggs and trehalose content. This standard curve allows for the enumeration of viable eggs in a sample in a fast and objective way. The only drawback of the method is that the concentration of viable eggs should not exceed 10.5 eggs/ $\mu$ l. This requires an estimation of the number of eggs so that an appropriate dilution of the egg suspension can be made prior to subsampling and subsequent measuring of trehalose content.

Although we did not observe fungal growth in the eggs or check for bacterial contamination, it should be noted that trehalose is the most widely distributed disaccharide in fungi (26) and can also be found in different classes of prokaryotes (2). Therefore, a positive signal might be possible from dead eggs because of fungal or bacterial contamination. In this case, trehalose from fungi or other sources can be released by sonication of samples for $120 \mathrm{~s}$ (12). This extracted trehalose is then degraded by trehalase and measured. Subsequently, the trehalose content of PCN eggs can be released by their incubation at $99^{\circ} \mathrm{C}$ for $30 \mathrm{~min}$.

Currently, a range of tests are used for assessing viability of eggs and $\mathrm{J} 2$ of plant-parasitic nematodes in different laboratories. Visual evaluation of the viability of eggs and $\mathbf{J} 2$ of both Globodera spp. is a simple means and recognized as a valid tool (18) for diagnostic laboratories. Visual assessment, nevertheless, is labor intensive and time consuming when many samples need to be evaluated; however, its largest disadvantage is that it requires well-trained and experienced personnel because it involves subjectivity. In our trial, the trehalose-based method gave results similar to those obtained with visual assessment. Meyer et al. (17) used different stains to estimate egg viability of Heterodera glycines. They concluded that some eggs could not be readily classified when using the stains and the results were not always consistent. Meldola's blue is the most popular and reliable staining agent to determine egg viability (15) and it has also been used to assess the egg viability of Globodera spp. when evaluating management measures (29) and conducting surveys of regulatory significance (22). Currently, Meldola's blue is not available for purchase.

Determination of viability by a hatching assay also requires a lot of time (weeks) and is subject to many factors such as diapause and the quality of the PRD (15). Our results showed that assessing the viability of both PCN species with a hatching assay lasting 10 weeks led to fewer live eggs than the other two methods. This slightly lower viability (about $2 \%$ ) obtained with the hatching assay could be attributed to some unhatched $\mathrm{J} 2$, which were viable even though they did not 
hatch. Perry and Moens (20) stated that a small percentage of J2 of $G$. rostochiensis do not hatch within a certain period due to diapause and persist in the field for many years before they hatch. An assessment of PNC viability for statutory purposes (field sampling for seed potato production; soil adhering to imported potato tubers) is usually needed in a short period of time. Hence, diagnostic labs performing hatching tests in less than 10 weeks can miss some live eggs still present inside cysts. Also, when viability is measured for large numbers of samples, the trehalose-based method is much faster than a longlasting hatching assay.

Compared with the existing viability methods, the trehalose-based method is a feasible, sensitive, and cheap technique. A single trehalose detection kit can be used for 1,000 to 10,000 viability tests and analyzing each sample costs $\$ 0.03$ to $\$ 0.27$, depending on the reaction volume. The trehalose-based method is also a standardized and objective technique requiring little training. This approach is likely to be a fast tool for assessing PCN egg viability when dealing with a large numbers of samples.

\section{Acknowledgments}

We thank H. Helder (Wageningen University) for his valuable technical advice. N. Ebrahimi thanks the Institute for Agricultural and Fisheries Research (ILVO) for financial support and Belgapom and Boerenbond for funding her Ph.D.

\section{Literature Cited}

1. Anonymous. 2007. Council Directive 2007/33/EC of 11 June 2007 on the Control of Potato Cyst Nematodes and Repealing Directive 69/465/EEC. Off. J. Eur. Union. http://eur-lex.europa.eu/legal-content/EN/TXT/?qid=1427640110255\&uri=CELEX: $32007 \mathrm{~L} 0033$

2. Arguelles, J. C. 2000. Physiological roles of trehalose in bacteria and yeasts: A comparative analysis. Arch. Microbiol. 174:217-224.

3. Brodie, B. B., and Mai, W. F. 1989. Control of the golden nematode in the United States. Annu. Rev. Phytopathol. 27:443-461.

4. Christoforou, M., Pantelides, I. S., Kanetis, L., Ioannou, N., and Tsaltas, D. 2014. Rapid detection and quantification of viable potato cyst nematodes using qPCR in combination with propidium monoazide. Plant Pathol. 63:1185-1192.

5. Clarke, A. J., and Hennessy, J. 1976. The distribution of carbohydrates in cysts of Heterodera rostochiensis. Nematologica 22:190-195.

6. Clarke, A. J., and Perry, R. N. 1977. Hatching of cyst nematodes. Nematologica 23:350-368.

7. Clarke, A. J., Perry, R. N., and Hennessy, J. 1978. Osmotic stress and the hatching of Globodera rostochiensis. Nematologica 24:384-392.

8. de Andrade, A. S. R., dos Santos, R. G., Nicoli, J. R., and Neves, M. J. 2000. Biochemical characterization of neutral trehalase activity in Saccharomyces boulardii. World J. Microbiol. Biotechnol. 16:691-694.

9. Deliopoulos, T., Devine, K. J., Haydock, P. P. J., and Jones, P. W. 2007. Studies on the effect of mycorrhization of potato roots on the hatching activity of potato root leachate towards the potato cyst nematodes, Globodera pallida and G. rostochiensis. Nematology 9:719-729.

10. Evans, K., and Rowe, J. A. 1998. Distribution and economic importance. Pages 1-30 in: The Cyst Nematodes. S. B. Sharma, ed. Kluwer Academic Publishers, Boston.
11. Hafez, S. L., Sundararaj, P., Handoo, Z. A., Skantar, A. M., Carta, L. K., and Chitwood, D. J. 2007. First report of the pale cyst nematode, Globodera pallida, in the United States. Plant Dis. 91:325.

12. Hallsworth, J. E., and Magan, N. 1994. Effects of carbohydrate type and concentration on polyhydroxy alcohol and trehalose content of conidia of three entomopathogenic fungi. Microbiology 140:2705-2713.

13. Hockland, S., Niere, B., Grenier, E., Blok, V., Phillips, M., Den Nijs, L., Anthoine, G., Pickup, J., and Viaene, N. 2012. An evaluation of the implications of virulence in non-European populations of Globodera pallida and G. rostochiensis for potato cultivation in Europe. Nematology 14:1-13.

14. Hodda, M., and Cook, D. C. 2009. Economic impact from unrestricted spread of potato cyst nematodes in Australia. Phytopathology 99:1387-1393.

15. Kroese, D., Zasada, I. A., and Ingham, R. E. 2011. Comparison of Meldola's Blue staining and hatching assay with potato root diffusate for assessment of Globodera sp. egg viability. J. Nematol. 43:182-186.

16. Marshall, J. W. 1998. Potato cyst nematodes (Globodera) species in New Zealand and Australia. Pages 353-394 in: Potato Cyst Nematodes, Biology, Distribution and Control. R. J. Marks and B. B. Brodie, eds. CAB International, Wallingford, UK.

17. Meyer, S. L. F., Sayre, R. M., and Huettel, R. N. 1988. Comparisons of selected stains for distinguishing between live and dead eggs of the plant-parasitic nematode Heterodera glycines. Proc. Helminthol. Soc. Wash. 55:132-139.

18. OEPP/EPPO. 2013. PM 7/40 (3) Globodera rostochiensis and Globodera pallida. Bull. OEPP/EPPO Bull. 43:119-138.

19. OEPP/EPPO. 2014. EPPO data sheets on quarantine pests. Online publication. http://www.eppo.int/QUARANTINE/nematodes/Globodera_pallida/ HETDSP_ds.pdf?utm_source=www.eppo.org\&utm_medium=int_redirect

20. Perry, R. N. 1989. Dormancy and hatching of nematode eggs. Parasitol. Today 5:377-383.

21. Perry, R. N., and Moens, M. 2011. Survival of parasitic nematodes outside the host. Pages 1-27 in: Molecular and Physiological Basis of Nematode Survival. R. N. Perry and D. A. Wharton, eds. CAB International, Oxford, UK.

22. Rott, M., Lawrence, T., Belton, M., Sun, F., and Kyle, D. 2010. Occurrence and detection of Globodera rostochiensis on Vancouver Island, British Columbia: An update. Plant Dis. 94:1367-1371.

23. Seinhorst, J. W. 1964. Methods for the extraction of Heterodera cysts from not previously dried soil samples. Nematologica 10:87-94.

24. Seinhorst, J. W. 1967. The relationships between population increase and population density in plant-parasitic nematodes. Nematologica 13:157-171.

25. Stanton, J. M. 1986. First record of potato cyst nematode, Globodera rostochiensis, in Australia. Australas. Plant Pathol. 15:87.

26. Thevelein, J. M. 1984. Regulation of trehalose mobilization in fungi. Microbiol. Rev. 48:42-59.

27. Turner, S. J., Fleming, C. C., Moreland, B. P., and Martin, T. J. G. 2009. Variation in hatch among pathotypes of the potato cyst nematodes, Globodera rostochiensis and $G$. pallida, in response to potato root diffusate from Solanum spp. I. Preliminary assessments to establish optimal testing conditions. Nematology 11:749-756.

28. Turner, S. L., and Subbotin, S. A. 2013. Cyst nematodes. Pages 109-143 in: Plant Nematology. R. N. Perry and M. Moens, eds. CAB International, Oxford.

29. Twomey, U., Warrior, P., Kerry, B. R., and Perry, R. N. 2000. Effects of the biological nematicide, DiTera, on hatching of Globodera rostochiensis and G. pallida. Nematology 2:355-362.

30. van den Elsen, S., Ave, M., Schoenmakers, N., Landeweert, R., Bakker, J., and Helder, J. 2012. A rapid, sensitive and cost-efficient assay to estimate viability of potato cyst nematodes. Phytopathology 102:140-146. 